\title{
A new precise date for the Tolmie Igneous Complex in northeastern Victoria
}

J. D. CLEMENS ${ }^{1 \star}$, D. FREI ${ }^{1}$ AND F. FINGER ${ }^{2}$

${ }^{1}$ Department of Earth Sciences, University of Stellenbosch, Private Bag X1, Matieland 7602, South Africa

${ }^{2}$ Department of Materials Engineering and Physics, University of Salzburg, Salzburg, Austria

*Corresponding author: jclemens@sun.ac.za

\section{SUPPLEMENTARY PAPER}

Australian Journal of Earth Sciences (2014) 61(7)
http://dx.doi.org/10.1080/08120099.2014.955058

Copies of Supplementary Papers may be obtained from the Geological Society of Australia's website (www.gsa.org.au), the Australian Journal of Earth Scienecs website (www.ajes.com.au) or from the National Library of Australia's Pandora archive (http://nla.gov.au/nla.arc-25194).

\section{SUPPLEMENTARY PAPERS}

Table A1 LA-SF-ICP-MS U-Pb dating methodology CAF, Stellenbosch University. Table A2 U-Pb age dating results for reference materials Plešovice and M127 obtained during this study by LA-SF-ICP-MS.

Table A3 U-Pb age dating results for sample Toom-1 obtained by LA-SF-ICP-MS. 
Table A1 LA-SF-ICP-MS U-Pb dating methodology CAF, Stellenbosch University

\begin{tabular}{|c|c|}
\hline \multicolumn{2}{|l|}{ Laboratory \& Sample Preparation } \\
\hline Laboratory name & Central Analytical Facility, Stellenbosch University \\
\hline Sample type / mineral & Igneous/inherited zircons \\
\hline Sample preparation & $\begin{array}{l}\text { Conventional mineral separation, } 1 \text { inch resin mount, } 1 \mu \mathrm{m} \text { polish to } \\
\text { finish (see text for more details) }\end{array}$ \\
\hline Imaging & CL, Zeiss Ultraplus (University of Salzburg) \\
\hline \multicolumn{2}{|l|}{ Laser ablation system } \\
\hline Make, Model \& type & Resonetics Resolution S155, ArF Excimer \\
\hline Ablation cell \& volume & Laurin Technology S155 double Helix large volume cell \\
\hline Laser wavelength & $193 \mathrm{~nm}$ \\
\hline Pulse width & $20 \mathrm{~ns}$ \\
\hline Fluence & Approx. $3 \mathrm{~J} / \mathrm{cm}^{-2}$ \\
\hline Repetition rate & $5.5 \mathrm{~Hz}$ \\
\hline Spot size & $30 \mu \mathrm{m}$ \\
\hline Sampling mode / pattern & $30 \mu \mathrm{m}$ single spot analyses \\
\hline Carrier gas & $\begin{array}{l}100 \% \mathrm{He}, \mathrm{Ar} \text { make-up gas injected into conical double Helix sampling } \\
\text { funnel }\end{array}$ \\
\hline $\begin{array}{l}\text { Pre-ablation laser warm-up } \\
\text { (background collection) }\end{array}$ & 3 cleaning shots followed by 20 seconds background collection \\
\hline Ablation duration & 20 seconds \\
\hline Wash-out delay & 15 seconds \\
\hline Cell carrier gas flow & $330 \mathrm{~mL} / \mathrm{min} \mathrm{He} \& 3.5 \mathrm{~mL} / \mathrm{min} \mathrm{N}_{2}$ \\
\hline \multicolumn{2}{|l|}{ ICP-MS Instrument } \\
\hline Make, Model \& type & Thermo Finnigan Element2 single collector HR-SF-ICP-MS \\
\hline Sample introduction & via polyurethane tubing \\
\hline RF power & $1350 \mathrm{~W}$ \\
\hline Make-up gas flow & $840 \mathrm{ml} / \mathrm{min} \mathrm{Ar}$ \\
\hline Detection system & Single collector secondary electron multiplier \\
\hline Masses measured & $202,204,206,207,208,232,233,235,238$ \\
\hline Integration time per peak & $4 \mathrm{~ms}$ \\
\hline Total integration time per reading & $1 \mathrm{sec}$ (represents the time resolution of the data) \\
\hline Sensitvity & $35000 \mathrm{cps} / \mathrm{ppm} \mathrm{Pb}$ \\
\hline Dead time & $6 \mathrm{~ns}$ \\
\hline \multicolumn{2}{|l|}{ Data Processing } \\
\hline Gas blank & 20 second on-peak \\
\hline Calibration strategy & $\begin{array}{l}\text { GJ-1 used as primary reference material, Plešovice \& M127 used as } \\
\text { secondary reference material (Quality Control) }\end{array}$ \\
\hline Reference Material info & $\begin{array}{l}\text { M127 (Nasdala et al. 2008; Mattinson 2010), Plešovice (Aftalion et al. } \\
\text { 1989; Slama et al. 2008), GJ-1 (Jackson et al. 2004) }\end{array}$ \\
\hline $\begin{array}{l}\text { Data processing package used / } \\
\text { Correction for LIEF* }\end{array}$ & $\begin{array}{l}\text { In-house spreadsheet data processing using intercept method for } \\
\text { LIEF correction }\end{array}$ \\
\hline Mass discrimination & $\begin{array}{l}\text { Standard-sample bracketing with }{ }^{207} \mathrm{~Pb} /{ }^{206} \mathrm{~Pb} \text { and }{ }^{206} \mathrm{~Pb} /{ }^{238} \mathrm{U} \\
\text { normalized to reference material } \mathrm{GJ}-1\end{array}$ \\
\hline $\begin{array}{l}\text { Common- } \mathrm{Pb} \text { correction, } \\
\text { composition and uncertainty }\end{array}$ & $\begin{array}{l}\text { 204-method, Stacey \& Kramers (1975) composition at the projected } \\
\text { age of the mineral, } 5 \% \text { uncertainty assigned }\end{array}$ \\
\hline Uncertainty level \& propagation & $\begin{array}{l}\text { Ages are quoted at } 2 \text { sigma absolute, propagation is by quadratic } \\
\text { addition. Reproducibility and age uncertainty of reference material anc } \\
\text { common-Pb composition uncertainty are propagated. }\end{array}$ \\
\hline Quality control / Validation & $\begin{array}{l}\text { Plešovice: Concordia age }=339 \pm 4(2 S D, M S W D=0.81, \text { Prob. }= \\
0.69) ; \text { M127: Concordia age }=529 \pm 4(2 S D, M S W D=0.09, \text { Prob. }= \\
\text { 1.00) }\end{array}$ \\
\hline Other information & For detailed method description see Frei \& Gerdes (2009) \\
\hline
\end{tabular}

*Laser-Induced Elemental Fractionation 
Table $4 \mathrm{U}-\mathrm{Pb}$ age dating results for sample Toom-1 obtained by LA-SF-ICP-MS

\section{RATIOS}

AGES [Ma]

Conc.

\begin{tabular}{|c|c|c|c|c|c|c|c|c|c|c|c|c|c|c|c|c|c|}
\hline Analysis & $\mathrm{U}[\mathrm{ppm}]^{\mathrm{a}}$ & $\mathrm{Pb}[p p m]^{\mathrm{a}}$ & $T h / U^{a}$ & ${ }^{207} \mathrm{~Pb} /{ }^{235} \mathrm{U}^{\mathrm{b}}$ & $2 \sigma^{d}$ & ${ }^{206} \mathrm{~Pb} /{ }^{238} \mathrm{U}^{\mathrm{b}}$ & $2 \sigma^{d}$ & rho $^{\mathrm{c}}$ & ${ }^{207} \mathrm{~Pb} /{ }^{206} \mathrm{~Pb}^{\mathrm{e}}$ & $2 \sigma^{d}$ & ${ }^{207} \mathrm{~Pb} /{ }^{235} \mathrm{U}$ & $2 \sigma$ & ${ }^{206} \mathrm{~Pb} /{ }^{238} \mathrm{U}$ & $2 \sigma$ & ${ }^{207} \mathrm{~Pb} /{ }^{206} \mathrm{~Pb}$ & $2 \sigma$ & $\%$ \\
\hline TO01_1C & 324 & 20 & 0.13 & 0.453 & 0.017 & 0.0605 & 0.0016 & 0.72 & 0.0543 & 0.0014 & 379 & 14 & 378 & 10 & 385 & 58 & 98 \\
\hline TO01_2 & 244 & 15 & 0.73 & 0.450 & 0.022 & 0.0602 & 0.0018 & 0.60 & 0.0543 & 0.0021 & 377 & 18 & 377 & 11 & 382 & 85 & 99 \\
\hline TO03_1C & 475 & 28 & 0.30 & 0.462 & 0.028 & 0.0596 & 0.0017 & 0.47 & 0.0563 & 0.0030 & 386 & 23 & 373 & 10 & 462 & 114 & 81 \\
\hline TO03_1R & 403 & 24 & 0.39 & 0.441 & 0.027 & 0.0592 & 0.0024 & 0.67 & 0.0540 & 0.0025 & 371 & 23 & 371 & 15 & 371 & 100 & 100 \\
\hline TO06_1R & 203 & 12 & 0.70 & 0.449 & 0.034 & 0.0599 & 0.0036 & 0.78 & 0.0543 & 0.0026 & 376 & 29 & 375 & 22 & 384 & 102 & 98 \\
\hline TO06_2 & 261 & 16 & 0.08 & 0.456 & 0.032 & 0.0606 & 0.0017 & 0.41 & 0.0546 & 0.0035 & 381 & 26 & 379 & 10 & 394 & 136 & 96 \\
\hline TO06_3 & 155 & 9 & 0.35 & 0.450 & 0.034 & 0.0601 & 0.0032 & 0.71 & 0.0543 & 0.0029 & 377 & 28 & 376 & 20 & 381 & 114 & 99 \\
\hline TO0̄ㄱ & 506 & 31 & 0.10 & 0.452 & 0.029 & 0.0604 & 0.0020 & 0.50 & 0.0543 & 0.0031 & 379 & 25 & 378 & 12 & 385 & 122 & 98 \\
\hline TO08_1C & 253 & 13 & 0.19 & 0.396 & 0.023 & 0.0530 & 0.0017 & 0.54 & 0.0542 & 0.0027 & 339 & 20 & 333 & 10 & 379 & 106 & 88 \\
\hline TO08_2 & 303 & 18 & 0.08 & 0.448 & 0.025 & 0.0599 & 0.0017 & 0.50 & 0.0542 & 0.0026 & 376 & 21 & 375 & 10 & 379 & 104 & 99 \\
\hline TO09_1C & 191 & 12 & 1.61 & 0.450 & 0.032 & 0.0603 & 0.0024 & 0.56 & 0.0541 & 0.0032 & 377 & 27 & 377 & 15 & 377 & 128 & 100 \\
\hline TO09_2C & 387 & 23 & 0.21 & 0.452 & 0.018 & 0.0604 & 0.0017 & 0.69 & 0.0543 & 0.0016 & 378 & 15 & 378 & 10 & 382 & 65 & 99 \\
\hline TO09_2R & 253 & 15 & 0.23 & 0.452 & 0.025 & 0.0605 & 0.0021 & 0.64 & 0.0542 & 0.0023 & 379 & 21 & 379 & 13 & 380 & 91 & 100 \\
\hline T010_1C & 309 & 19 & 0.27 & 0.451 & 0.034 & 0.0606 & 0.0032 & 0.70 & 0.0540 & 0.0030 & 378 & 29 & 379 & 19 & 371 & 118 & 102 \\
\hline TO10_2 & 214 & 13 & 0.23 & 0.444 & 0.033 & 0.0596 & 0.0031 & 0.70 & 0.0541 & 0.0028 & 373 & 28 & 373 & 19 & 373 & 113 & 100 \\
\hline TO11_1 & 210 & 13 & 1.54 & 0.507 & 0.028 & 0.0604 & 0.0016 & 0.49 & 0.0609 & 0.0030 & 416 & 23 & 378 & 10 & 636 & 102 & 59 \\
\hline T011_2 & 261 & 16 & 3.05 & 0.546 & 0.028 & 0.0607 & 0.0017 & 0.54 & 0.0653 & 0.0028 & 442 & 23 & 380 & 10 & 783 & 88 & 48 \\
\hline T011_3 & 159 & 10 & 1.48 & 0.450 & 0.024 & 0.0602 & 0.0018 & 0.56 & 0.0542 & 0.0024 & 378 & 20 & 377 & 11 & 381 & 95 & 99 \\
\hline TO11_4 & 115 & 7 & 1.40 & 0.800 & 0.038 & 0.0602 & 0.0021 & 0.75 & 0.0965 & 0.0030 & 597 & 28 & 377 & 13 & 1558 & 57 & 24 \\
\hline T013_2C & 177 & 11 & 0.96 & 0.466 & 0.029 & 0.0604 & 0.0018 & 0.48 & 0.0560 & 0.0030 & 388 & 24 & 378 & 11 & 450 & 116 & 84 \\
\hline TO13_2R & 330 & 16 & 1.75 & 0.396 & 0.023 & 0.0493 & 0.0021 & 0.74 & 0.0582 & 0.0023 & 339 & 20 & 310 & 13 & 537 & 85 & 58 \\
\hline TO1̄5 & 186 & 11 & 0.98 & 0.451 & 0.023 & 0.0604 & 0.0019 & 0.62 & 0.0542 & 0.0022 & 378 & 19 & 378 & 12 & 378 & 88 & 100 \\
\hline TO15C & 152 & 9 & 1.03 & 0.452 & 0.025 & 0.0603 & 0.0022 & 0.65 & 0.0543 & 0.0023 & 378 & 21 & 378 & 14 & 383 & 92 & 98 \\
\hline TO15R & 143 & 8 & 0.84 & 0.419 & 0.039 & 0.0550 & 0.0019 & 0.37 & 0.0552 & 0.0048 & 355 & 33 & 345 & 11 & 421 & 182 & 82 \\
\hline T016 & 316 & 19 & 0.08 & 0.458 & 0.024 & 0.0610 & 0.0018 & 0.56 & 0.0544 & 0.0023 & 383 & 20 & 382 & 11 & 389 & 93 & 98 \\
\hline T017C & 262 & 14 & 0.24 & 0.408 & 0.018 & 0.0547 & 0.0014 & 0.59 & 0.0541 & 0.0020 & 347 & 16 & 344 & 9 & 373 & 80 & 92 \\
\hline TO17R & 173 & 10 & 0.22 & 0.452 & 0.023 & 0.0603 & 0.0022 & 0.73 & 0.0544 & 0.0019 & 379 & 19 & 377 & 14 & 385 & 76 & 98 \\
\hline T018_1 & 203 & 12 & 0.30 & 0.451 & 0.020 & 0.0604 & 0.0017 & 0.63 & 0.0542 & 0.0019 & 378 & 17 & 378 & 10 & 379 & 75 & 100 \\
\hline T018_2 & 154 & 9 & 3.02 & 0.452 & 0.024 & 0.0603 & 0.0019 & 0.59 & 0.0544 & 0.0024 & 378 & 20 & 377 & 12 & 386 & 94 & 98 \\
\hline TO1̄̄ & 211 & 13 & 0.65 & 0.451 & 0.026 & 0.0602 & 0.0017 & 0.49 & 0.0543 & 0.0027 & 378 & 22 & 377 & 11 & 383 & 109 & 98 \\
\hline TO19C & 183 & 11 & 0.98 & 0.450 & 0.022 & 0.0603 & 0.0019 & 0.64 & 0.0541 & 0.0020 & 377 & 18 & 378 & 12 & 374 & 82 & 101 \\
\hline TO19R & 70 & 4 & 1.04 & 0.448 & 0.045 & 0.0603 & 0.0024 & 0.39 & 0.0539 & 0.0050 & 376 & 38 & 378 & 15 & 366 & 195 & 103 \\
\hline
\end{tabular}

${ }^{\mathrm{a}} \mathrm{U}$ and $\mathrm{Pb}$ concentrations and Th/U ratios are calculated relative to $\mathrm{GJ}-1$ reference zircon. ${ }^{\mathrm{b}}$ Corrected for background and within-run $\mathrm{Pb} / \mathrm{U}$ fractionation and normalised to reference zircon $\mathrm{GJ}-1$ (ID-TIMS values/measured value); ${ }^{207} \mathrm{~Pb} /{ }^{235} \mathrm{U}$ calculated using $\left({ }^{207} \mathrm{~Pb} /{ }^{206} \mathrm{~Pb}\right) /\left({ }^{238} \mathrm{U} /{ }^{206} \mathrm{~Pb} * 1 / 137.88\right)$. ${ }^{\circ} \mathrm{Rho}$ is the error correlation defined as the quotient of the propagated errors of the ${ }^{206} \mathrm{~Pb} /{ }^{238} \mathrm{U}$ and the ${ }^{207} \mathrm{~Pb} /{ }^{235} \mathrm{U}$ ratio. ${ }^{\mathrm{d}}$ Quadratic addition of within-run errors $(2 \mathrm{SD})$ and daily reproducibility of GJ-1 (2 SD). ${ }^{e}$ Corrected for mass-bias by normalising to GJ-1 reference zircon ( $\sim 0.6$ per atomic mass unit) and common $\mathrm{Pb}$ using the model $\mathrm{Pb}$ composition of Stacey \& Kramers (1975). 
Table A3 U-Pb age dating results for reference materials Plešovice and M127 obtained during this study by LA-SF-ICP-MS

\begin{tabular}{|c|c|c|c|c|c|c|c|c|c|c|c|c|c|c|c|c|c|c|}
\hline \multirow[b]{2}{*}{ Sample } & \multirow[b]{2}{*}{ Analysis } & \multirow[b]{2}{*}{$U$ [ppm] $]^{a}$} & \multirow[b]{2}{*}{$\mathrm{Pb}[p p m]^{\mathrm{a}}$} & \multirow[b]{2}{*}{$\mathrm{Th} / \mathrm{U}^{\mathrm{a}}$} & \multicolumn{7}{|c|}{ RATIOS } & \multicolumn{6}{|c|}{ AGES [Ma] } & \multirow{2}{*}{$\begin{array}{c}\text { Conc. } \\
\%\end{array}$} \\
\hline & & & & & ${ }^{207} \mathrm{~Pb} /{ }^{235} \mathrm{U}^{\mathrm{b}}$ & $2 \sigma^{d}$ & ${ }^{206} \mathrm{~Pb} / /^{238} \mathrm{U}^{\mathrm{b}}$ & $2 \sigma^{d}$ & rhoc & ${ }^{207} \mathrm{~Pb} /{ }^{206} \mathrm{~Pb}^{\mathrm{e}}$ & $2 \sigma^{d}$ & ${ }^{207} \mathrm{~Pb} /{ }^{235} \mathrm{U}$ & $2 \sigma$ & ${ }^{206} \mathrm{~Pb} /{ }^{238} \mathrm{U}$ & $2 \sigma$ & ${ }^{207} \mathrm{~Pb} /{ }^{206} \mathrm{~Pb}$ & $2 \sigma$ & \\
\hline Plešovice & PL_001 & 851 & 46 & 0.12 & 0.395 & 0.011 & 0.0540 & 0.0011 & 0.76 & 0.0531 & 0.0009 & 338 & 9 & 339 & 7 & 335 & 39 & 101 \\
\hline Plešovice & PL_002 & 877 & 47 & 0.12 & 0.393 & 0.011 & 0.0539 & 0.0011 & 0.76 & 0.0528 & 0.0009 & 336 & 9 & 339 & 7 & 320 & 39 & 106 \\
\hline Plešovice & PL_003 & 856 & 46 & 0.12 & 0.386 & 0.010 & 0.0543 & 0.0011 & 0.76 & 0.0517 & 0.0009 & 332 & 9 & 341 & 7 & 270 & 40 & 126 \\
\hline Plešovice & PL_004 & 854 & 46 & 0.12 & 0.395 & 0.011 & 0.0538 & 0.0011 & 0.75 & 0.0532 & 0.0010 & 338 & 9 & 338 & 7 & 336 & 41 & 101 \\
\hline Plešovice & PL_005 & 786 & 42 & 0.12 & 0.393 & 0.012 & 0.0536 & 0.0011 & 0.71 & 0.0531 & 0.0011 & 336 & 10 & 337 & 7 & 333 & 47 & 101 \\
\hline Plešovice & PL_006 & 860 & 46 & 0.12 & 0.400 & 0.012 & 0.0541 & 0.0011 & 0.72 & 0.0537 & 0.0011 & 342 & 10 & 339 & 7 & 357 & 45 & 95 \\
\hline Plešovice & PL_007 & 965 & 52 & 0.12 & 0.396 & 0.013 & 0.0539 & 0.0011 & 0.64 & 0.0533 & 0.0013 & 339 & 11 & 338 & 7 & 341 & 56 & 99 \\
\hline Plešovice & PL_008 & 687 & 37 & 0.09 & 0.400 & 0.015 & 0.0542 & 0.0012 & 0.59 & 0.0536 & 0.0017 & 342 & 13 & 340 & 8 & 353 & 68 & 96 \\
\hline Plešovice & PL_009 & 575 & 31 & 0.09 & 0.398 & 0.014 & 0.0543 & 0.0013 & 0.66 & 0.0532 & 0.0014 & 340 & 12 & 341 & 8 & 337 & 59 & 101 \\
\hline Plešovice & PL_010 & 406 & 22 & 0.07 & 0.393 & 0.015 & 0.0541 & 0.0013 & 0.62 & 0.0527 & 0.0016 & 336 & 13 & 339 & 8 & 315 & 67 & 108 \\
\hline M127 & 127_001 & 841 & 72 & 0.39 & 0.685 & 0.017 & 0.0858 & 0.0017 & 0.79 & 0.0579 & 0.0009 & 530 & 13 & 531 & 10 & 526 & 33 & 101 \\
\hline M127 & 127_002 & 834 & 71 & 0.39 & 0.685 & 0.017 & 0.0857 & 0.0017 & 0.79 & 0.0579 & 0.0009 & 530 & 13 & 530 & 10 & 527 & 34 & 101 \\
\hline M127 & 127_003 & 813 & 70 & 0.39 & 0.687 & 0.017 & 0.0860 & 0.0017 & 0.78 & 0.0579 & 0.0009 & 531 & 13 & 532 & 10 & 526 & 34 & 101 \\
\hline M127 & 127_004 & 877 & 75 & 0.40 & 0.686 & 0.019 & 0.0855 & 0.0017 & 0.74 & 0.0581 & 0.0011 & 530 & 15 & 529 & 10 & 535 & 41 & 99 \\
\hline M127 & 127_005 & 802 & 69 & 0.39 & 0.683 & 0.018 & 0.0857 & 0.0017 & 0.76 & 0.0578 & 0.0010 & 529 & 14 & 530 & 10 & 524 & 37 & 101 \\
\hline M127 & 127_006 & 838 & 71 & 0.40 & 0.680 & 0.019 & 0.0850 & 0.0017 & 0.75 & 0.0581 & 0.0011 & 527 & 14 & 526 & 10 & 532 & 40 & 99 \\
\hline M127 & 127_007 & 816 & 70 & 0.40 & 0.683 & 0.019 & 0.0856 & 0.0018 & 0.75 & 0.0579 & 0.0011 & 529 & 15 & 529 & 11 & 526 & 40 & 101 \\
\hline
\end{tabular}

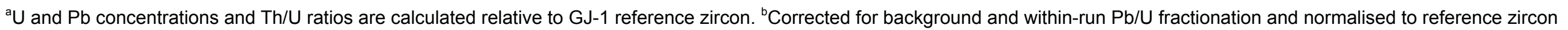

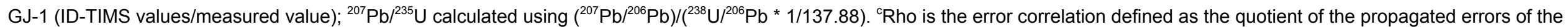

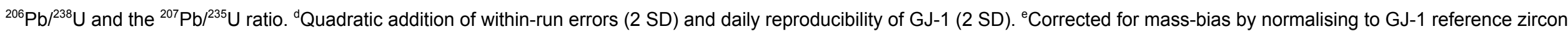

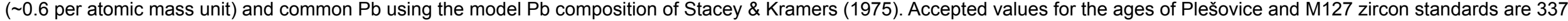
\pm 1 (Slama et al. 2008) and $524 \pm 2 \mathrm{Ma}$ (Klötzli et al. 2009), respectively. 


\section{REFERENCES}

Aftalion M., Bowes D. R. \& VŔana S. 1989. Early Carboniferous U-Pb zircon ages for garnetiferous perpotassic granulites, Blanský les massif, Czechoslovakia. Neues Jahrbuch für Mineralogie, Monatshefte 4, 145-152.

FreI D. \& GeRDES A. 2009. Precise and accurate in situ U-Pb dating of zircon with high sample throughput by automated LA-SF-ICPMS. Chemical Geology 261, 261-270. doi: 10.1016/j.chemgeo.2008.07.025

Jackson S., Pearson N. J., Griffin W. L. \& Belousova E. A. 2004. The application of laser ablation - inductively coupled plasma - mass spectrometry to in situ U$\mathrm{Pb}$ zircon geochronology. Chemical Geology 211, 47-69. doi: 10.1016/j.chemgeo.2004.06.017

KLÖTZLI U., KLÖTZLI E., GÜNES Z. \& KOSLER J. 2009. Accuracy of laser ablation U-Pb zircon dating: results from a test using five different reference zircons. Geostandards and Analytical Research 33, 5-15.

MATtinson J. M. 2010. Analysis of the relative decay constants of ${ }^{235} U$ and ${ }^{238} U$ by multi-step CA-TIMS measurements of closed-system natural zircon samples. Chemical Geology 275, 186-198. doi: 10.1016/j.chemgeo.2010.05.007

Nasdala L., Hofmeister W., Norberg N., Mattinson J. M., Corfu F., Dörr W., Kamo S. L., Kennedy A. K., Kronz A., Reiners P. W., Frei D., Košler J., Wan Y., GötZe J., HÄGer T., KRÖner A. \& VAlley J. W. 2008. Zircon M257-a homogeneous natural reference material for the ion microprobe $\mathrm{U}-\mathrm{Pb}$ analysis of zircon. Geostandards and Geoanalytical Research 32, 247-265. doi: 10.1111/j.1751-908X.914.X

Sláma J., Košler J., Condon D. J., Crowley J. L., Gerdes A., Hanchar J. M., Horstwood M. S. A., Morris G. A., Nasdala L., Norberg N., Schaltegger U., Schoene B., Tubrett M. N. \& Whitehouse M. J. 2008. Plešovice zircon-a new natural reference material for $\mathrm{U}-\mathrm{Pb}$ and $\mathrm{Hf}$ isotopic microanalysis. Chemical Geology 249, 1-35. doi: 10.1016/j.chemgeo.2007.11.005

StACEY J. S. \& KrameRS J. D. 1975. Approximation of terrestrial lead isotope evolution by a two-stage model. Earth and Planetary Science Letters 26, 207221. doi: 10.1016/0012-821X 